\title{
Supercapacitor with superior electrochemical properties derived from symmetrical manganese oxide-carbon fiber coated with polypyrrole
}

\begin{abstract}
A supercapacitor electrode comprising conducting polypyrrole (PPy) coated on manganese oxide-carbon fiber (CNFMnO2) was successfully synthesized using electrospinning, followed by carbonization and in-situ polymerization. A non-uniform distribution of PPy on the surface of CNFMnO2 was observed via FESEM analysis. The chemical bonding of CNFMnO2/PPy and the valence state of manganese were revealed via FTIR, Raman spectroscopy, XRD and XPS measurements. CNFMnO2/PPy composite possessed high specific capacitance and specific energy of $315.80 \mathrm{Fg}-1$ and $13.68 \mathrm{Wh} / \mathrm{kg}$, respectively. In addition, good electrochemical reversibility was proven upon CNFMnO2/PPy even at higher sweep rate (5$200 \mathrm{mV} / \mathrm{s}$ ). Moreover, this one-dimensional electrode achieved an excellent long-term cycling stability $(82.46 \%)$ over $2000 \mathrm{CV}$ cycles with low charge transfer resistance $(4.61 \Omega)$. The modification of CNFMnO2/PPy contributes to good synergistic effects among the material which improve the electrochemical behavior of manganese oxide-based fiber composite for future supercapacitor.
\end{abstract}

Keyword: Polyacrylonitrile; Manganese oxide; Carbon fiber; Polypyrrole; Supercapacitor 